\title{
Deprojecting the quenching of star formation in and near clusters
}

\author{
G. A. Mamon, S. Mahajan and S. Raychaudhury
}

\begin{abstract}
Using $\mathrm{H}_{\delta}$ and $\mathrm{D}_{n} 4000$ as tracers of recent or ongoing efficient star formation, we analyze the fraction of SDSS galaxies with recent or ongoing efficient star formation (GORES) in the vicinity of 268 clusters. We confirm the well-known segregation of star formation, and using Abel deprojection, we find that the fraction of GORES increases linearly with physical radius and then saturates. Moreover, we find that the fraction of GORES is modulated by the absolute line-of-sight velocity (ALOSV): at all projected radii, higher fractions of GORES are found in higher ALOSV galaxies. We model this velocity modulation of GORES fraction using the particles in a hydrodynamical cosmological simulation, which we classify into virialized, infalling and backsplash according to their position in radial phase space at $z=0$. Our simplest model, where the GORES fraction is only a function of class does not produce an adequate fit to our observed GORES fraction in projected phase space. On the other hand, assuming that in each class the fraction of GORES rises linearly and then saturates, we are able to find well-fitting 3D models of the fractions of GORES. In our best-fitting models, in comparison with $18 \%$ in the virial cone and $13 \%$ in the virial sphere, GORES respectively account for 34\% and 19\% of the infalling and backsplash galaxies, and as much as $11 \%$ of the virialized galaxies, possibly as a result of tidally induced star formation from galaxy-galaxy interactions. At the virial radius, the fraction of GORES of the backsplash population is much closer to that of the virialized population than to that of the infalling galaxies. This suggests that the quenching of efficient star formation is nearly complete in a single passage through the cluster.
\end{abstract}

\section{Introduction}

It is well known that the cluster environment affects the physical properties of galaxies, as there is a segregation with projected radius of morphology [7], color (e.g. [3]), luminosity (e.g. [1]) and spectral indices such as the equivalent width of [OII] or $\mathrm{H}_{\alpha}$ (e.g. [2; 81]). At the same time, there are indications of velocity segregation of luminosity [4] and of star formation efficiency: emission-line galaxies tend to span a wider dispersion of velocities than galaxies without such lines [5]. In fact, it was

G. A. Mamon

IAP (UMR 7095: CNRS \& UPMC), Paris, France, e-mail: gameiap.fr

S. Mahajan \& S. Raychaudhury

School of Physics \& Astronomy, Univ. of Birmingham, UK 
long known that spiral galaxies in clusters span a wider distribution of velocities than ellipticals and S0s (e.g. [13]). However, those spirals without emission lines and that are not morphologically disturbed span the same velocity distribution as the early type galaxies [12]. Relative to passive galaxies (without $\mathrm{H}_{\alpha}$ in emission), the trend for higher velocity dispersions of galaxies with emission $\left(\mathrm{H}_{\alpha}\right)$ lines found by [5] is reversed outside the virial radius [14].

In the present work [10], we take advantage of the large statistics of the Sloan Digital Sky Survey (SDSS) to better quantify the velocity modulation of the radial segregation of the diagnostics of star formation efficiency. We then model the SDSS observed fractions of Galaxies with Ongoing or Recent Efficient Star Formation (GORES), with the help of a cosmological simulation.

\section{Observed Velocity Modulation}

We select clusters of at least 15 galaxies from the SDSS-DR4 group catalog of [16], with criteria $0.02 \leq z \leq 0.12, M_{180}>10^{14} M_{\odot}$ and with at least $12 M_{r}<-20.5$ galaxies within $R_{180}$. Around these 268 clusters, we select galaxies in a slightly wider redshift range, lying within $2 R_{100}=2.6 R_{180}$ from the cluster in projected space, with absolute line-of-sight velocity relative to the cluster mean (ALOSV) within $3 \sigma_{v}$, with absolute magnitude $M_{r}<-20.5$, and with angular effective radius $\theta_{\text {eff }} \leq 5^{\prime \prime}$ to avoid large galaxies for which the SDSS fibers only span the inner region. This selection yields 19904 galaxies and we also select 21000 field galaxies that lie at least $10 \mathrm{Mpc}$ in projection away from any cluster (corresponding to typically $6.7 R_{\mathrm{v}}$, where the virial radius $R_{\mathrm{v}} \equiv R_{100}$ ).

We identify GORES as galaxies with both $\mathrm{H}_{\delta}>2 \AA$ and $\mathrm{D}_{n} 4000<1.5$ (age less than $1 \mathrm{Gyr}$ for most galaxies, [9]). The symbols in the left panel of Fig. 11illustrate how the observed fraction of GORES increases with projected radius. More important, this plot shows that this radial segregation of GORES fraction is modulated by ALOSV: there are significantly more (fewer) GORES among the higher (lower) ALOSV galaxies.

\section{Deprojection}

We first deproject the fraction of GORES without taking into account their ALOSVs. Writing $g_{\text {GORES }}(R)=N_{\text {GORES }}(R) / N_{\text {tot }}(R)$, we can deduce the surface densities, $\Sigma(R)=N(R) /(2 \pi R)$ of GORES and of all galaxies, and then perform Abel deprojection yielding space densities $v(r)=-(1 / \pi) \int_{R}^{\infty} \Sigma^{\prime}(R) d R / \sqrt{R^{2}-r^{2}}$ for both GORES and all galaxies. The right panel of Fig. 11 shows that this deprojection leads to a fraction of GORES that rises linearly with physical radius and then saturates as

$$
f_{\mathrm{GORES}}(r)=f_{0} \frac{r}{r+a},
$$



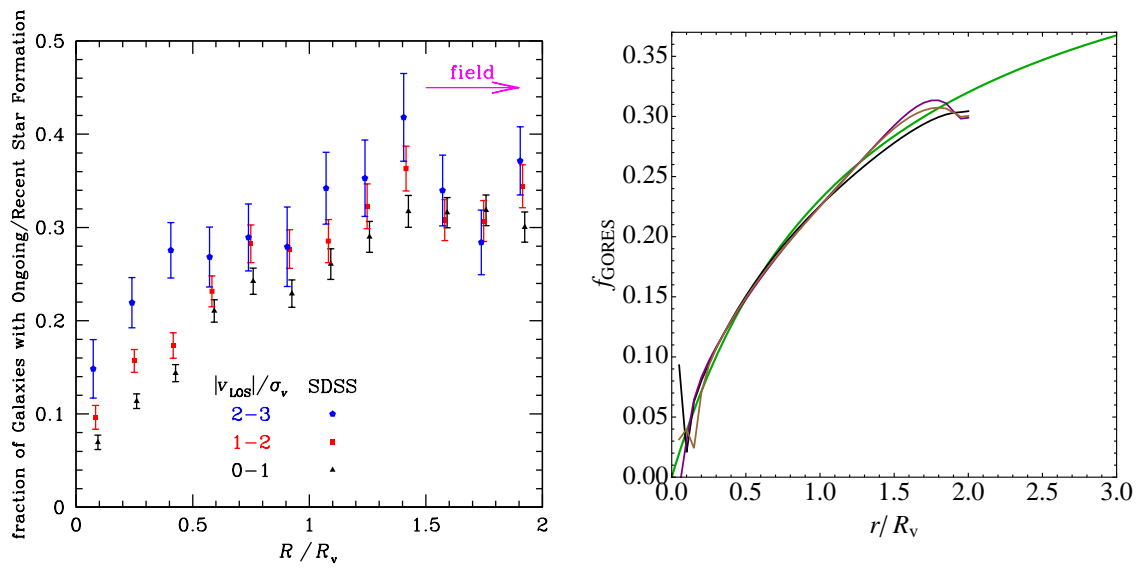

Fig. 1 Left: Observed fraction of GORES in three bins of ALOSV. Right: Deprojected fractions of GORES. Thin curves show polynomial fits of the $\log$ surface density vs. $\log R$ (orders 3,4 , and 5). The longer thick curve is Eq. 11 with $f_{0}=0.52$ and $a=1.26 R_{\mathrm{v}}$, obtained by a $\chi^{2}$ fit to the order 4 polynomial fit of $\log \Sigma$ vs. $\log R$ with $r$ linearly spaced between 0.05 and $2 R_{\mathrm{v}}$.

with best-fit parameters of $f_{0}=0.52$ and $a=1.26 R_{\mathrm{v}}$.

We now assume that the observed fraction of GORES in projected phase space, $g_{\mathrm{GORES}}\left(R,\left|v_{\mathrm{LOS}}\right|\right)$, is also a fraction of the dynamical class of galaxies: we distinguish virialized, infalling and backsplash galaxies according to their $z=0$ position in radial phase space in a cosmological simulation as shown in the left panel of Fig. 2. The right panel of Fig. 2 shows that in our standard scheme, where particles in regions A and B are both infalling (this scheme provides the best fits to the observed fraction of GORES in projected phase space), the fraction of backsplash particles reaches over 50\% just beyond the virial radius and for the lowest ALOSVs.

We suppose that the fraction of GORES varies with physical radius $r$ as in Eq. (1), varying the normalization and possibly the scale for each class:

$$
f_{\alpha}(r)=f_{\alpha} \frac{r / R_{\mathrm{v}}}{r / R_{\mathrm{v}}+a_{\alpha}}
$$

i.e. rising roughly linearly with radius for $r<a_{\alpha}$, saturating to an asymptotic value $f_{\alpha}$ at large radii. The predicted fractions of GORES is then

$$
g_{\mathrm{GORES}}\left(R_{i}, v_{j}\right)=\sum_{\alpha} p\left(\alpha \mid R_{i}, v_{j}\right) \sum_{k} f_{\alpha}\left(r_{k}\right) q\left(r_{k} \mid R_{i}, v_{j}, \alpha\right)
$$

where $p\left(\alpha \mid R_{i}, v_{j}\right)$ is the probability that a particle located at projected radius $R_{i}$ and ALOSV $v_{j}$ is of class $\alpha$, while $q\left(k \mid R_{i}, v_{j}, \alpha\right)$ is the fraction of the particles of class $\alpha$ in the cell of projected phase space $\left(R_{i}, v_{j}\right)$ that are in the $k$ th bin of physical radius (with $r_{k}=R_{i} \cosh u_{k}$, where $u_{k}$ is linearly spaced from 0 to $\cosh ^{-1} r_{\max } / R_{i}$, using $r_{\max }=50 R_{\mathrm{v}}$ ). We measure $p$ and $q$ from the dark matter particles in the stack of 93 regular mock clusters [11] in a hydrodynamical cosmological simulation [6]. 

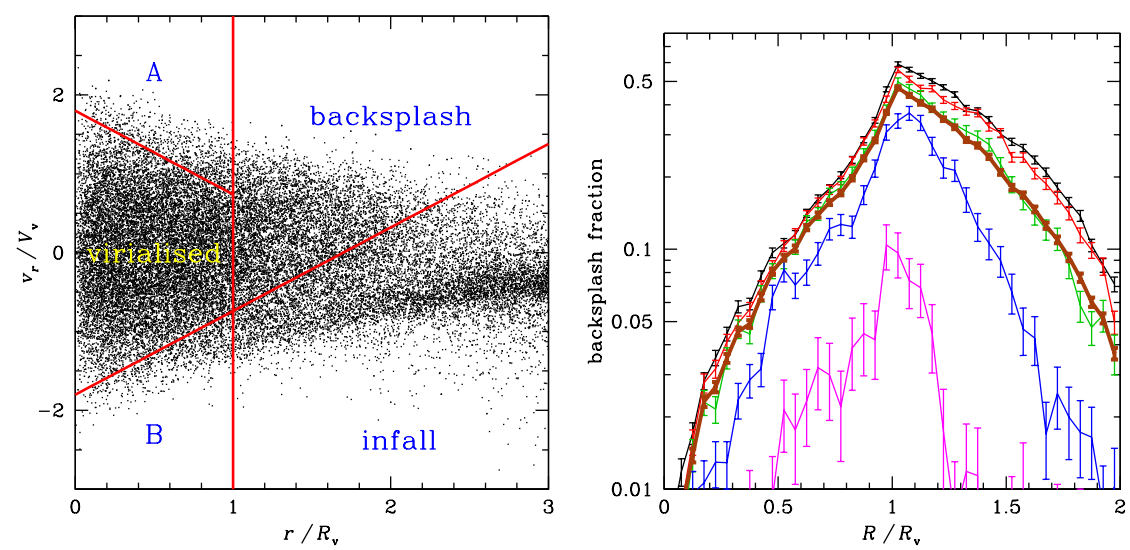

Fig. 2 Left: Radial phase space distribution (in virial units) of dark matter particles of a stack of 93 mock regular mock clusters from a cosmological hydrodynamical simulation [6]. The critical velocity separating infall from backsplash population (long diagonal line) is taken from a single halo of an older, dissipationless, cosmological simulation [15]. For clarity, only 1 out of 550 particles of the original simulation is plotted. The letters indicate uncertain classes. Right: Fraction of backsplash particles in projected phase space, for our standard scheme, particles in regions A and $\mathrm{B}$ are infalling. The thin lines indicate ALOSV bins increasing from $0.25,0.75, \ldots, 1.75$, and $>2 \sigma_{v}$, going from top to bottom, while the thick line shows the average.
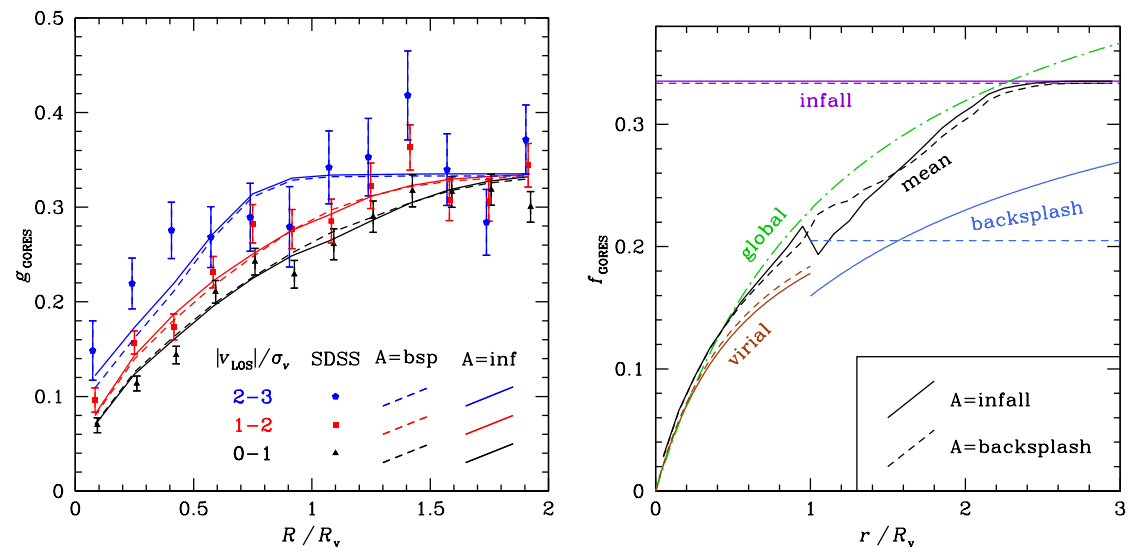

Fig. 3 Left: Observed fraction of GORES with two best-fitting models (Eq. [2] using Eq. [3]) overplotted, both assume that the particles in B are of the infalling class. Right: Deprojected fraction of GORES for the two best-fitting models.

Fig. 3 shows how the best-fitting models for the two best fitting schemes (right panel, both with $\mathrm{B}=$ infall, but with $\mathrm{A}=$ infall and $\mathrm{A}=$ backsplash, respectively leading to $\chi^{2}=1.2$ and 1.4 per degree of freedom with cosmic variance from the simulation included) fit the observed fraction of GORES in projection (left panel). Schemes 
where regions $\mathrm{A}$ and $\mathrm{B}$ are both virialized or both backsplash, or where $f_{\mathrm{GORES}}$ is constant per class produce substantially worse fits.

Interestingly, the two best-fitting models yield a GORES fraction that is independent of radius for the infall population. Moreover, in the best fitting scheme, the galaxies bouncing out of the cluster with very high positive line-of-sight velocities are of the infalling class. This suggests that the quenching of efficient star formation is not instantaneous as the infalling galaxies pass through the pericenter of their orbit. Moreover, inspection of the right panel of Fig. 3 indicates that at $r=R_{\mathrm{v}}$, where the three galaxy classes can be compared, the backsplash GORES fraction is much closer to that of the virialized class than to the infall GORES fraction (in fact in our best fitting scheme $\mathrm{A}=$ infall, the backsplash fraction of GORES is lower than the corresponding fraction for the virial class, but forcing equal fractions of GORES for the two classes at the virial radius produces an equally good fit). This suggests that as galaxies cross through clusters, efficient star formation is nearly completely quenched once galaxies reach the virial sphere, either after the first pericenter on their way out, or at least before they are mixed with the virialized galaxies.

While GORES account for $18 \%$ of the galaxies within the virial cone, in our best fitting models, they also account for 13\% within the virial sphere, 34\% and 19\% of the infalling and backsplash classes, respectively. GORES also account for as many as $11 \%$ of the virialized galaxies, perhaps the consequence of star formation resulting from tides caused by interactions with other galaxies.

\section{References}

[1] C. Adami, A. Biviano, \& A. Mazure, 1998, A\&A, 331, 439

[2] M. Balogh, V. Eke, C. Miller, et al., 2004, MNRAS, 348, 1355

[3] M. L. Balogh, I. K. Baldry, R. Nichol, et al., 2004, ApJ, 615, L101

[4] A. Biviano, M. Girardi, G. Giuricin, F. Mardirossian, \& M. Mezzetti, 1992, ApJ, 396, 35

[5] A. Biviano, P. Katgert, A. Mazure, et al., 1997, A\&A, 321, 84

[6] S. Borgani, G. Murante, V. Springel, et al., 2004, MNRAS, 348, 1078

[7] A. Dressler, 1980, ApJ, 236, 351

[8] C. P. Haines, F. La Barbera, A. Mercurio, P. Merluzzi, \& G. Busarello, 2006, ApJ, 647, L21

[9] G. Kauffmann, T. Heckman, S. D. M. White, et al., 2003, MNRAS, 341, 33

[10] S. Mahajan, G. A. Mamon, \& S. Raychaudhury, 2011, MNRAS, submitted

[11] G. A. Mamon, A. Biviano, \& G. Murante, 2010, A\&A, 520, A30

[12] C. Moss, 2006, MNRAS, 373, 167

[13] C. Moss \& R. J. Dickens, 1977, MNRAS, 178, 701

[14] K. Rines, M. J. Geller, M. J. Kurtz, \& A. Diaferio, 2005, AJ, 130, 1482

[15] T. Sanchis, E. Łokas, \& G. A. Mamon, 2004, A\&A, 414, 445

[16] X. Yang, H. J. Mo, F. C. van den Bosch, et al., 2007, ApJ, 671, 153 